\title{
Successful thrombolytic therapy in acute ischemic stroke after reversal of warfarin: a case report
}

\author{
Gang Qiu ${ }^{1 \#}$, Yi-Dan Yan ${ }^{2 \#}$, Xue-Feng Zhang ${ }^{1 \#}$, Ming Shen ${ }^{1}$, Jiao Qian ${ }^{3}$, Xiao-Dong Ma ${ }^{1}$, Zhi-Chun Gu ${ }^{2}$ \\ ${ }^{1}$ Department of Pharmacy, Department of Critical Medicine, Medical Department, Department of Neurology, Haiyan People's Hospital, Haiyan, \\ China; ${ }^{2}$ Department of Pharmacy, Renji Hospital, School of Medicine, Shanghai Jiaotong University, Shanghai, China; ${ }^{3}$ Department of Pharmacy, \\ Changhai Hospital, Second Military Medical University, Shanghai, China \\ \#These authors contributed equally to this work. \\ Correspondence to: Jiao Qian, MD. Department of Pharmacy, Changhai Hospital, Second Military Medical University, Shanghai 200433 , China. \\ Email: qianjiao@smmu.edu.cn; Xiao-Dong Ma. Department of Neurology, Haiyan People's Hospital, Haiyan 314300, China. \\ Email: haiyan120120@126.com.
}

\begin{abstract}
It is essential for acute ischemic stroke (AIS) patients to receive timely revascularization. However, intravenous thrombolysis (IVT) is not recommended for AIS patients with warfarin associated hypocoagulability. Meanwhile, monotherapy of coagulation factors or vitamin $\mathrm{K}$ is unable to reverse anticoagulation of warfarin in emergency. Thus, developing an effective IVT strategy poses a challenging task for these fragile population. Herein, an 82-year-old male, on regular administration with warfarin because of nonvalvular atrial fibrillation (NVAF), suffered from AIS and had an elevated international normalized ratio value of 1.72 and prolonged prothrombin time of $18.2 \mathrm{~s}$ at stroke onset. For normalizing INR, combination of 4 factor prothrombin complex concentrate, fresh frozen plasma and vitamin $\mathrm{K}_{1}$ were administrated. Finally, the patient successfully received recombinant tissue plasminogen activator (rt-PA), with an obviously neurological improvement. This case shows a feasible role of IVT therapy with rt-PA after reversal of coagulation regarding AIS patients with warfarin-related hypocoagulability.
\end{abstract}

Keywords: Thrombolytic therapy; ischemic stroke; warfarin; atrial fibrillation; case report

Submitted Apr 01, 2020. Accepted for publication Jul 21, 2020.

doi: 10.21037/apm-20-868

View this article at: http://dx.doi.org/10.21037/apm-20-868

\section{Introduction}

Warfarin is used to prevent ischemic stroke (IS) in patients with atrial fibrillation (AF). However, the annual acute ischemic stroke (AIS) risk is approximately 1.7 percent for warfarin-treated $\mathrm{AF}$ patients, occurring despite $30 \%$ of them having an international normalized ratio (INR) within the therapeutic range (2.5-3.5) (1). Timely revascularization is essential for AIS patients, while intravenous thrombolysis (IVT) is not recommended for patients with INR $>1.7$ or/ and prothrombin time $(\mathrm{PT})>15 \mathrm{~s}$ because of increased risk of bleeding (2). In addition, traditional reversal strategy with vitamin $\mathrm{K}$ is hardly to normalize INR rapidly. Goldstein et al. revealed that rapid INR reduction (INR $\leq 1.3$ at $30 \mathrm{~min}$ after the end of infusion) was achieved in $55 \%$ (48/87) patients using vitamin $\mathrm{K}$ and 4-factor prothrombin complex concentrate (4F-PCC) compared with $10 \%$ $(8 / 81)$ of patients receiving vitamin $\mathrm{K}$ and fresh frozen plasma (FFP). Insufficient normalization of INR inevitably limits the application of IVT, making a poor outcome in these patients (3). For such challenging task, combined utilization with 4F-PCC, FFP and vitamin K may represent a reasonable alternative regarding the improvement of time to INR normalization. Here, we report a nonvalvular atrial fibrillation (NVAF) patient under warfarin treatment who suffered from AIS and had an elevated INR and prolonged PT at stroke onset. Finally, thrombolytic therapy was successfully applied after reversal of warfarin in present patient. We present the following article in accordance with the CARE reporting checklist (available at http://dx.doi. org/10.21037/apm-20-868). 

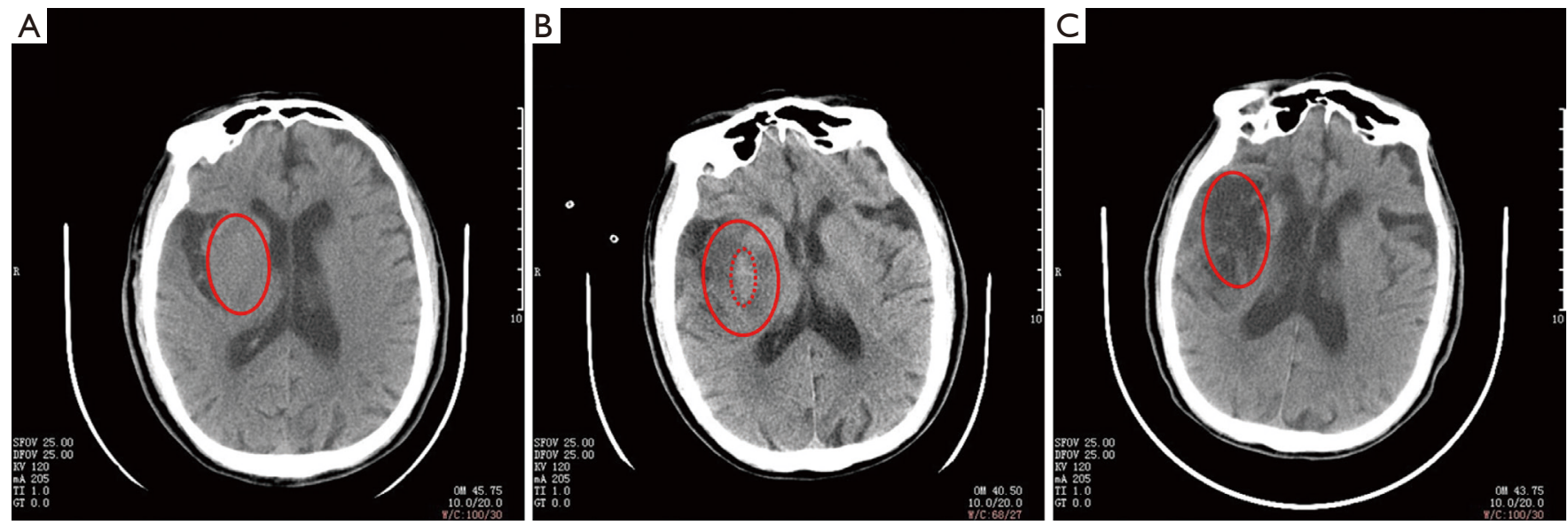

Figure 1 Images imported to our emergency center upon admission. Head computed tomography (CT) imaging of the supply area of right basal ganglia prior to reversal of warfarin (A; area with red solid line); CT imaging 24 hours after IVT revealed acute infarction (B; area with red solid line) and mild hemorrhagic transformation of parenchymal hematoma type 1 (B; area with red dotted line); CT imaging one year after IVT showed a low-density area due to old ischemic stroke (C; area with red solid line).

\section{Case presentation}

An 82-year-old male (weight of $57 \mathrm{~kg}$ ) was admitted to our emergency department within 90 minutes after experiencing sudden onset of dysarthria and left-sided hemiparesis on Mar 10, 2017, with an initial National Institutes of Health Stroke Scale (NIHSS) score of 40 (very severe stroke), indicating a poor prognosis and necessity for thrombolytic or mechanical intervention. Head computed tomography (CT) was performed immediately after arrival in the hospital (Figure 1A). Before AIS, he was receiving warfarin at the dosage of $3.75 \mathrm{mg}$ daily for more than 10 years due to NVAF, and had a history of AIS 10 years ago but without severe sequelae. For the present patient, $\mathrm{CHA}_{2} \mathrm{DS}_{2}-\mathrm{VAS}_{\mathrm{C}}$ score, which is recommended for assessment of stroke risk in patients with $\mathrm{AF}$, was 4 and indicated a high stroke risk. After admission, his blood examination showed an elevated INR value of 1.72 (reference: $0.80-1.25$ ) and prolonged PT of $18.2 \mathrm{~s}$ (reference: 9.40-12.50 s), precluding IVT. Besides, mechanical thrombectomy was unavailable in our center. Thus, reversal of anticoagulation was considered before receiving IVT therapy with recombinant tissue plasminogen activator (rt-PA). For normalizing INR, 4 factor prothrombin complex concentrate (4F-PCC) $200 \mathrm{IU}$, fresh frozen plasma (FFP) $260 \mathrm{~mL}$ and vitamin $\mathrm{K}_{1} 10 \mathrm{mg}$ were administrated by slow infusion. Thirty minutes later, the patient's coagulation was improved accompanied with reductive INR value of 1.5 and PT of $16.8 \mathrm{~s}$. Given that the PT was still longer than $15 \mathrm{~s}$, additional $120 \mathrm{~mL}$ of FFP was administrated. For IVT, an intravenous bolus of $5.13 \mathrm{mg}$ of rt-PA (51.3 mg totally, $0.9 \mathrm{mg} / \mathrm{kg}$ ) was administered intravenously at 159 minutes after the onset of symptoms, followed by remaining $46.17 \mathrm{mg}$ via continuously intravenous infusion within 1 hour. After 24 hours of the thrombolysis (Mar 11, 2017), the patient's NIHSS score markedly improved from 40 to 33 , and the CT scan showed a mild hemorrhagic transformation of parenchymal hematoma $(\mathrm{PH})$ type 1 (Figure $1 \mathrm{~B}$ ). Warfarin at the dosage of $3.75 \mathrm{mg}$ resumed on day 14 post-IVT (Mar 24, 2017) and the patient's NIHSS score was gradually improved to 11 during 3-month follow-up (Jun 15, 2017). A CT scan one year later (Mar 15, 2018) showed a low-density area due to old ischemic stroke without hemorrhage (Figure 1C). Timeline of the treatment and follow-up in this patient is presented in Table 1. All procedures performed in studies involving human participants were in accordance with the ethical standards of the institutional and/or national research committee(s) and with the Helsinki Declaration (as revised in 2013). Written informed consent was obtained from the patient for publication of this manuscript and any accompanying images.

\section{Discussion}

Atrial fibrillation is the most common arrhythmia, with a prevalence of approximately $1 \%$ among the adult population (4). Patients with NVAF, due to their 
Table 1 Timeline of the treatment and follow-up in the present patient

\begin{tabular}{lll}
\hline Time course & Date & Events \\
\hline Stroke onset & Mar 10, 2017 & Dysarthria and left-sided hemiparesis \\
90 minutes & Mar 10, 2017 & Admitted to emergency department (NIHSS score: 40; INR: 1.72; PT: 18.2 s) \\
110 minutes & Mar 10, 2017 & Reversal of anticoagulation: 4F-PCC 200 IU, FFP 380 mL, vitamin K1 10 mg \\
159 minutes & Mar 10, 2017 & IVT: rt-PA 51.3 mg \\
24 hours after IVT & Mar 11, 2017 & Mild hemorrhagic transformation; NIHSS score: 33 \\
14 days & Mar 24, 2017 & Warfarin resumed (3.75 mg) \\
3 months & Jun 15, 2017 & NIHSS score: 11 \\
12 months & Mar 15, 2018 & CT scan showed a low-density area due to old ischemic stroke without hemorrhage \\
\hline
\end{tabular}

NIHSS, National Institutes of Health Stroke Scale; INR, international normalized ratio; PT, prothrombin time; 4F-PCC, 4-factor prothrombin complex concentrate; FFP, fresh frozen plasma; IVT, intravenous thrombolysis; rt-PA, recombinant tissue plasminogen activator; CT, computed tomography.

pathological hypercoagulability, have a 5 -fold increased risk of ischemic stroke relative to those without NVAF (5). According to a meta-analysis for NVAF patients (6), adjusted-dose of warfarin was associated with a $64 \%$ reduction in stroke, and the absolute risk reduction in all strokes was $2.7 \%$ per year for primary prevention and $8.4 \%$ per year for secondary prevention. Given above reasons, warfarin as well as direct oral anticoagulants (DOACs) have been generally used for stroke prevention in patients with NVAF. However, the annual AIS risk remains about $1.7 \%$ in warfarin-treated NVAF patients (1). It is of note that direct IVT is not recommended for AIS patients with INR $>1.7$ or/and prothrombin time (PT) $>15 \mathrm{~s}$ because of increased risk of bleeding (2). For these fragile population, the reversal strategy of warfarin-related coagulation is pivotal. Sanák et al. showed that standard IVT underwent within a 3-hour time window from stroke onset had clinical benefit and did not increase the risk of cerebral hemorrhage in patients with AF and AIS (7). Therefore, consideration also needs to be given to the importance of thrombolytic timing.

The common method of supplementation of coagulation factors (4F-PCC, FFP) in combination with vitamin $\mathrm{K}$ is used to fast reversal of warfarin in such clinical setting. (I) Certainly, administration of vitamin $\mathrm{K}$ is the most straightforward intervention to counteract the effect of warfarin $(8,9)$. However, the value of INR starts to drop within 4 hours of vitamin $\mathrm{K}$ infusion and will be normalized after $12-16$ hours. Thus, monotherapy of vitamin $\mathrm{K}$ is unable to reverse anticoagulation of warfarin in emergency; (II) FFP, contained a low concentration of factors II, VII, IX and $\mathrm{X}$, was used to be the most common coagulation factor replacement product for rapid reversal of warfarin in North America $(10,11)$. The use of FFP also has some limitations, including the risk of volume overload and transfusion reactions, as well as insufficient normalization of INR value $(10,11)$; (III) as for 4F-PCC, it contains seven coagulation factors (factors II, VII, IX, X, protein C and protein S) and causes rapid reversal of warfarin-induced coagulation (12). 4F-PCC has been available in urgent reversal of warfarin therapy for decades in Europe, while it was not approved until 2008 in Canada and 2013 in the USA, mainly due to the uncertainty of prothrombotic complications. Recently, a meaningful meta-analysis study has been conducted on the focus of this issue by pooling nineteen studies with 2,878 patients and demonstrated that 4F-PCC is an effective and safe option in reversal of warfarin associated bleeding episodes (13). Chausson et al. also suggested that reversal strategy of 4F-PCC bolus and vitamin K before IVT was feasible and safe in AIS patients under warfarin with INR $>1.7$, without increasing the risk for intracerebral bleeding or other complications (14). Summarily, combination of vitamin K, PCC and FFP is likely to be an alternative with a good balance between efficacy and safety in AIS patients with hypocoagulability.

In the present AIS patient, his significantly elevated INR and prolonged PT at initial blood examination was immediately normalized with the combination of vitamin K, PCC and FFP. Since the thrombolysis was timely carried out within 3 hours from our patient's onset of stroke, sufficient dosage of rt-PA $(0.9 \mathrm{mg} / \mathrm{kg})$ was administrated, resulting in a satisfactory outcome. We believe that this case report provides useful information regarding IVT strategy 
in AIS patients with warfarin-associated hypocoagulability. However, no RCTs have been conducted to evaluate the reversal strategy for these patients, thus further high-quality real-world studies on evaluation of this issue are necessary.

Based on the clinical setting of this case and current evidence, IVT therapy with rt-PA after reversal of warfarin may represent feasible and safe regarding AIS patients with warfarin-related hypocoagulability.

\section{Acknowledgments}

Funding: This study was supported by medical and health research project of Zhejiang province (2018KY811), Research Funds of Shanghai Health and Family Planning commission (20184Y0022), Cultivation fund of clinical research of Renji Hospital (PY2018-III-06), and Clinical Pharmacy Innovation Research Institute of Shanghai Jiao Tong University School of Medicine (CXYJY2019ZD001, CXYJY2019QN004).

\section{Footnote}

Reporting Checklist: The authors have completed the CARE reporting checklist. Available at http://dx.doi.org/10.21037/ apm-20-868

Peer Review File: Available at http://dx.doi.org/10.21037/ apm-20-868

Conflicts of Interest: All authors have completed the ICMJE uniform disclosure form (available at http://dx.doi. org/10.21037/apm-20-868). The authors have no conflicts of interest to declare.

Ethical Statement: The authors are accountable for all aspects of the work in ensuring that questions related to the accuracy or integrity of any part of the work are appropriately investigated and resolved. All procedures performed in studies involving human participants were in accordance with the ethical standards of the institutional and/or national research committee(s) and with the Helsinki Declaration (as revised in 2013). Written informed consent was obtained from the patient for publication of this manuscript and any accompanying images.

Open Access Statement: This is an Open Access article distributed in accordance with the Creative Commons Attribution-NonCommercial-NoDerivs 4.0 International
License (CC BY-NC-ND 4.0), which permits the noncommercial replication and distribution of the article with the strict proviso that no changes or edits are made and the original work is properly cited (including links to both the formal publication through the relevant DOI and the license). See: https://creativecommons.org/licenses/by-nc-nd/4.0/.

\section{References}

1. Prabhakaran S, Rivolta J, Vieira JR, et al. Symptomatic intracerebral hemorrhage among eligible warfarin-treated patients receiving intravenous tissue plasminogen activator for acute ischemic stroke. Arch Neurol 2010;67:559-63.

2. Powers WJ, Rabinstein AA, Ackerson T, et al. 2018 Guidelines for the Early Management of Patients With Acute Ischemic Stroke: A Guideline for Healthcare Professionals From the American Heart Association/ American Stroke Association. Stroke 2018;49:e46-e110.

3. Goldstein JN, Refaai MA, Milling TJ Jr, et al. Fourfactor prothrombin complex concentrate versus plasma for rapid vitamin $\mathrm{K}$ antagonist reversal in patients needing urgent surgical or invasive interventions: a phase $3 \mathrm{~b}$, open-label, non-inferiority, randomised trial. Lancet 2015;385:2077-87.

4. Feinberg WM, Blackshear JL, Laupacis A, et al. Prevalence, age distribution, and gender of patients with atrial fibrillation: analysis and implications. Arch Intern Med 1995;155:469-73.

5. Wolf PA, Abbott RD, Kannel WB. Atrial fibrillation: a major contributor to stroke in the elderly: the Framingham study. Arch Intern Med 1987;147:1561-64.

6. Hart RG, Pearce LA, Aguilar MI. Meta-analysis: Antithrombotic Therapy to Prevent Stroke in Patients Who Have Nonvalvular Atrial Fibrillation. Ann Intern Med 2007;146:857-67.

7. Sanák D, Herzig R, Král M, et al. Is atrial fibrillation associated with poor outcome after thrombolysis? J Neurol 2010;257:999-1003.

8. Crowther MA, Ageno W, Garcia D, et al. Oral vitamin $\mathrm{K}$ versus placebo to correct excessive anticoagulation in patients receiving warfarin: A randomized trial. Ann Intern Med 2009;150:293-300.

9. Keeling D, Baglin T, Tait C, et al. Guidelines on oral anticoagulation with warfarin -fourth edition. $\mathrm{Br} \mathrm{J}$ Haematol 2011;154:311-24.

10. Bechtel BF, Nunez TC, Lyon JA, et al. Treatments for reversing warfarin anticoagulation in patients with acute intracranial hemorrhage: a structured literature review. Int 
J Emerg Med 2011;4:40.

11. Ageno W, Gallus AS, Wittkowsky A, et al. Oral anticoagulant therapy: Antithrombotic Therapy and Prevention of Thrombosis, 9th ed: American College of Chest Physicians Evidence-Based Clinical Practice Guidelines. Chest 2012;141:e44S-e88S.

12. Leissinger CA, Blatt PM, Hoots WK, et al. Role of prothrombin complex concentrates in reversing warfarin anticoagulation: A review of the literature. Am J Hematol

Cite this article as: Qiu G, Yan YD, Zhang XF, Shen M, Qian J, Ma XD, Gu ZC. Successful thrombolytic therapy in acute ischemic stroke after reversal of warfarin: a case report. Ann Palliat Med 2021;10(5):5813-5817. doi: 10.21037/apm-20-868
2008;83:137-43.

13. Brekelmans MPA, Ginkel KV, Daams JG, et al. Benefits and harms of 4-factor prothrombin complex concentrate for reversal of vitamin $\mathrm{K}$ antagonist associated bleeding: a systematic review and meta-analysis. J Thromb Thrombolysis 2017;44:118-29.

14. Chausson N, Soumah D, Aghasaryan M, et al. Reversal of Vitamin K Antagonist Therapy Before Thrombolysis for Acute Ischemic Stroke. Stroke 2018;49:2526-8. 